\title{
THE GEODESIC FLOW FOR DISCRETE GROUPS OF INFINITE VOLUME
}

\author{
PETER J. NICHOLLS ${ }^{1}$
}

\begin{abstract}
Let $\Gamma$ be a discrete group acting in the unit ball $B$ of euclidean $n$-space and $T(B)$ the unit tangent space of $B$. We define the geodesic flow $g_{t}$ on the quotient space $\Omega=T(B) / \Gamma$ and show that for discrete groups of infinite volume the flow is of zero type-namely, for measurable subsets $A, B$ of $\Omega$ which are of finite measure, $\lim _{t \rightarrow \infty} g_{t}(A) \cap B=0$. Using this result, we give a new and elementary proof of the fact that for a discrete group of infinite volume, $N(r)=$ $o(V\{x:|x|<r\})$ as $r \rightarrow 1$, where $N(r)$ is the orbital counting function and $V$ denotes hyperbolic volume.
\end{abstract}

1. Introduction. Let $B$ denote the unit ball $\{x:|x|<1\}$ of $R^{n}$ with the Poincaré metric defined by the element $d s=|d x| /\left(1-|x|^{2}\right)$. Let $G$ denote the full group of Moebius transformations which preserve $B$ and hence also the sphere $S=\{x$ : $|x|=1\}$. If $\Gamma$ is a discrete subgroup of $G$ which contains no element fixing the origin (except the identity), we may define the Dirichlet region $D$ for $\Gamma$ by

$$
D=\{x \in B: s(x, 0)<s(\gamma(x), 0) \text { all } \gamma \in \Gamma, \gamma \neq I\}
$$

The volume $V$ derived from

$$
d V=2^{n}\left(1-|x|^{2}\right)^{-n} d x_{1} d x_{2} \cdots d x_{n},
$$

where $x=\left(x_{1}, x_{2}, \ldots, x_{n}\right)$, is invariant under Moebius transformations preserving $B$. We say the group $\Gamma$ is of finite volume if $V(D)<\infty$, and of infinite volume otherwise.

Consider now the unit tangent space $T(B)$ of $B$. The points of $T(B)$ consist of a point $x$ of $B$ and a direction at that point. The direction is given by a unit vector $\xi$ of $S$. Thus $T(B)$ is $B \times S$ and is thus equipped with the product topology. The Moebius group $G$ acts on $T(B)$ as follows. For $\gamma$ in $G$ we denote the Jacobian matrix of $\gamma$ at $x$ by $\gamma^{\prime}(x)$. This matrix is a positive multiple of an orthogonal matrix and this multiple, which measures the change of linear scale, will be denoted by $\left|\gamma^{\prime}(x)\right|$. We then have

$$
\gamma(x, \xi)=\left(\gamma(x),\left(\gamma^{\prime}(x) /\left|\gamma^{\prime}(x)\right|\right) \xi\right) .
$$

The invariant volume element is $d m=d V d w(\xi)$, where $w(\xi)$ denotes the solid angle.

Received by the editors May 9, 1984 and, in revised form, February 15, 1985.

1980 Mathematics Subject Classification. Primary 30F40; Secondary $20 \mathrm{H} 10$.

${ }^{1}$ Research supported in part by N.S.F. grant no. MCS 8102393.

(C)1986 American Mathematical Society $0002-9939 / 86 \$ 1.00+\$ .25$ per page 
Every element $(x, \xi)$ of $T(B)$ determines a geodesic ray which starts from $x$ in the direction $\xi$. For a real number $t$ let $x$ move along the geodesic form $x$ to a point $x^{\prime}$ at a directed hyperbolic distance $t$ from $x$. At the same time let $\xi$ slide to the positive tangent vector $\xi^{\prime}$ at $x^{\prime}$. We define the geodesic flow $g_{t}$ on $T(B)$ by

$$
g_{t}(x, \xi)=\left(x^{\prime}, \xi^{\prime}\right)
$$

It is quite evident that $g_{t} \circ g_{s}=g_{t+s},\left(g_{t}\right)^{-1}=g_{-t}$, and that for any Moebius transform $\gamma$,

$$
g_{t} \circ \gamma=\gamma \circ g_{t}
$$

It can be shown that $g_{t}$ leaves invariant the volume element $d m[1, \mathrm{p} .76]$. In view of these comments we see that for any discrete group $\Gamma$, the flow $g_{t}$ is defined on the quotient space $\Omega=T(B) / \Gamma$ and preserves there the measure $m$ inherited from the measure $m$ on $T(B)$.

Clearly the space $\Omega$ has finite $(m)$ measure if and only if the underlying group $\Gamma$ is of finite volume, and in this case Hopf showed in 1939 [2, p. 291] that the geodesic flow is mixing. His result is as follows (where $P$ denotes a point of $\Omega$ ).

THEOREM A. If $\Gamma$ is a discrete group of finite volume and if $f, \lambda$ are bounded integrable functions on $\Omega$, then

$$
\lim _{t \rightarrow \infty} \int_{\Omega} f\left(g_{-t}(P)\right) \lambda(P) d m=\int_{\Omega} f(P) d m \int_{\Omega} \lambda(P) d m .
$$

In a recent paper [7] Shirakawa considers the case $n=2$ ( $\Gamma$ is a Fuchsian group), where $\Gamma$ is of infinite area, and proves the following result.

THEOREM B. If $\Gamma$ is a Fuchsian group of infinite area and if the geodesic flow is ergodic on $\Omega$, then it is of zero type. Namely, for $f, \lambda$ bounded integrable functions on $\Omega$,

$$
\lim _{t \rightarrow \infty} \int_{\Omega} f\left(g_{-t}(P)\right) \lambda(P) d m=0 .
$$

Our aim in this paper is to generalize this result to all dimensions and to remove the condition that $g_{t}$ be ergodic. We will prove

THEOREM 1. If $\Gamma$ is a discrete group of infinite volume, then, for $f, \lambda$ bounded integrable functions on $\Omega$,

$$
\lim _{t \rightarrow \infty} \int_{\Omega} f\left(g_{-t}(P)\right) \lambda(P) d m=0 .
$$

This result may be used to provide a new proof of the asymptotic estimate for the orbital counting function in the infinite volume case (Patterson [6] for $n=2$, Nicholls [4] for $n \geqslant 2$ ). Defining, for $0<r<1, N(r)$ to be the cardinality of the set $\{\gamma \in \Gamma:|\gamma(0)|<r\}$, we have

THEOREM 2. If $\Gamma$ is a discrete group of infinite volume, then

$$
N(r)=o(V\{x:|x|<r\}) \text { as } r \rightarrow 1 \text {. }
$$


It should be remarked here that the present proof of this theorem avoids all the technical details of the previous proof [4] and is also at a much more elementary level since it does not use Sullivan's deep result on the ergodicity of the geodesic flow.

The theorems are proved in the next section.

2. Proofs of Theorems 1 and 2. The proofs are given in three parts. We first show that for any discrete group $\Gamma$ the conclusions of Theorems 1 and 2 are equivalent. Next we show that Theorem 2 is true for any group of convergence type, and, finally, it is shown that Theorem 1 is true for a group $\Gamma$ of infinite volume and divergence type.

Let $\Delta$ be a ball centered at the origin and contained in $D$. With $w$ as before denoting solid angle subtended at the origin, we note that the properties

$$
N(r)=o(V\{x:|x|<r\}) \text { as } r \rightarrow 1,
$$

and

$$
w(\Gamma(\Delta) \cap\{x:|x|=r\})=o(1) \text { as } r \rightarrow 1
$$

are equivalent. This is proved in two dimensions in [3, Theorem 5] and the proof generalizes easily to higher dimensions. We define

$$
h(r)=w(\Gamma(\Delta) \cap\{x:|x|=r\}) .
$$

The author has previously shown [5, Lemma 3.1] that $h$ is uniformly continuous for $0<r<1$.

Now let $A$, a subset of $T(B)$, be the ball $\Delta$, together with all directions at each point; thus $A=\Delta \times S$. If $\Delta$ is of noneuclidean radius $\delta$, then $g_{t}(A)$ is the "annular region" in $B,\{x: t-\delta<s(x, 0)<t+\delta\}$, together with a set of directions $I(x)$ at each point. It is geometrically evident that $w(I(x))$ depends only on $r(=|x|)$. We may thus write $w(I(x))=B(r)$, and so

$$
m\left(g_{t}(A) \cap \Gamma(A)\right)=\int_{a}^{b} 2^{n} h(r) B(r)\left(1-r^{2}\right)^{-n} d r
$$

where $a=\left(e^{t-\delta}-1\right) /\left(e^{t-\delta}+1\right)$ and $b=\left(e^{t+\delta}-1\right) /\left(e^{t+\delta}+1\right)$. Thus $s(0, a)=t$ $-\delta$ and $s(0, b)=t+\delta$. Noting that $m(A)=m\left(g_{t}(A)\right)$, which is equal to

$$
\int_{a}^{b} 2^{n} w(S) B(r)\left(1-r^{2}\right)^{-n} d r
$$

we see that condition (2), i.e., $h(r)=o(1)$ as $r \rightarrow 1$, implies that

$$
m\left(g_{t}(A) \cap \Gamma(A)\right)=o(m(A))=o(1)
$$

as $t \rightarrow \infty$. The converse also holds by the uniform continuity of $h$, and so we have shown that condition (2) is equivalent to

$$
\lim _{t \rightarrow \infty} m\left(g_{t}(A) \cap \Gamma(A)\right)=0 .
$$

If we assume the conclusion of Theorem 1 and let $f=\lambda$ be the characteristic function of the projection of $A$ on $\Omega$, then (3) follows immediately. 
Now we show that (3) implies the conclusion of Theorem 1. By conjugation, (3) will be true for any set $A$ derived from a small ball $\Delta$ and thus for any open set in $T(B)$ of finite noneuclidean measure. Interpreting this result on the quotient space $\Omega$, we see that for any open subset $U$ of $\Omega$ with $m(U)<\infty$,

$$
\lim _{t \rightarrow \infty} m\left(g_{t}(U) \cap U\right)=0 .
$$

Now if $X$ is a measurable subset of $\Omega$ with $m(X)<\infty$, then, since $X$ is a subset of an open set of finite measure, we have

$$
\lim _{t \rightarrow \infty} m\left(g_{t}(X) \cap X\right)=0
$$

from which it follows that for $X, Y$ of finite measure in $\Omega$,

$$
\lim _{t \rightarrow \infty} m\left(g_{t}(X) \cap Y\right)=0
$$

By considering characteristic functions, we see that this statement is equivalent to the conclusion of Theorem 1. Thus the conclusions of Theorems 1 and 2 are equivalent.

The group $\Gamma$ is said to be of convergence type if the series $\sum_{\gamma \in \Gamma}(1-|\gamma(0)|)^{n-1}$ converges. In this case the integral $\int_{0}^{1}(1-t)^{n-1} d N(t)$ and, hence, also the integral $\int_{0}^{1}(1-t)^{n-2} N(t) d t$ converges, it follows from this that $\int_{r}^{(1+r) / 2}(1-t)^{n-2} N(t) d t$ approaches zero as $r \rightarrow 1$. So, using the fact that $N(t)$ is increasing, we have that $(1-r)^{n-1} N(r) \rightarrow 0$ as $r \rightarrow 1$. An easy calculation shows that

$$
V\{x:|x|<r\} \sim k(n) /(1-r)^{n-1} \text { as } r \rightarrow 1,
$$

and so Theorem 2 is proved in the case that $\Gamma$ is of convergence type.

It remains therefore to prove Theorem 1 under the hypotheses that $\Gamma$ is of divergence type and of infinite volume. Our method of proof follows the work of Hopf [2], in which he uses the connnection between the geodesic and horocyclic flows to establish his mixing result-Theorem A. We wish to extend Hopf's method to the infinite volume case and to avoid using the ergodicity of the geodesic flow.

In order to define a horocycle in the $n$-dimensional setting $(n>2)$, it is easier to consider the upper half-space model of hyperbolic space $-R^{n-1} \times(0, \infty)$-in which a horocycle at $\infty$ through the point $y$ is a set of vectors of the form $y+W$, where $W$ is a one-dimensional subspace of $R^{n-1}$. If $x$ is in $B$ and $\xi$ is in $S$, we may define a horocycle at $\xi$ through $x$ by conjugating to the upper half-space, sending $\xi$ to $\infty$ and $x$ to $y$. An element of $T(B)$ does not determine a unique horocycle-in order to do this we need a point and two directions. Thus we define the biped space $U(B)$ to be the set of triples $(x, \alpha, \beta)$ where $x$ is in $B$ and $\alpha, \beta$ are orthogonal unit vectors of $S$. A point of $U(B)$ determines a unique horocycle; to see how this is done, consider $b=(x, \alpha, \beta)$ and conjugate to the upper half-space by a Moebius transform, $V$ say, so that $V(b)=\left(y, \alpha_{1}, \beta_{1}\right)$ with $\alpha_{1}$ in the direction of the $x_{n}$-axis. It follows that $\beta_{1}$ is in $R^{n-1}$, and we have a horocycle $y+\left\langle\beta_{1}\right\rangle$, where $\left\langle\beta_{1}\right\rangle$ denotes all multiples of $\beta_{1}$. The $V^{-1}$ image of this horocycle is a horocycle in $B$ through $x$ at $\xi$, where $\xi$ is the endpoint of the geodesic determined by the element $(x, \alpha)$ of $T(B)$.

The Moebius group acts on $U(B)$ in the natural way:

$$
\gamma(x, \alpha, \beta)=\left(\gamma(x),\left(\gamma^{\prime}(x) /\left|\gamma^{\prime}(x)\right|\right) \alpha,\left(\gamma^{\prime}(x) /\left|\gamma^{\prime}(x)\right|\right) \beta\right) .
$$


The invariant volume element is

$$
d \mu=d V d w(\alpha) d v(\beta)
$$

where $v(\beta)$ denotes the solid angle at the origin subtended from the $(n-2)$ dimensional unit sphere of directions orthogonal to a given $\alpha$.

The horocyclic flow, $h_{s}$, is defined on $U(B)$ as follows. Given $b=(x, \alpha, \beta)$ and $s$ real, first determine the horocycle from $b$ and move $x$ along the horocycle a directed hyperbolic distance $s$ to arrive at a point $y$, at the same time sliding $\beta$ around the horocycle to a new direction $\beta^{\prime}$ at $y$. Clearly there is a unique vector $\alpha^{\prime}$ such that $(x, \alpha, \beta)$ and $\left(y, \alpha^{\prime}, \beta^{\prime}\right)$ determine the same horocycle, and we set

$$
h_{s}(x, \alpha, \beta)=\left(y, \alpha^{\prime}, \beta^{\prime}\right) \text {. }
$$

We now wish to define the geodesic flow $g_{t}$ on $U(B)$. In view of our previous definition of $g_{t}$ on $T(B)$, it is quite clear that we should have

$$
g_{t}(x, \alpha, \beta)=\left(g_{t}(x, \alpha), \theta\right),
$$

where $g_{t}$ on the left is the action on $U(B)$, and on the right is the action on $T(B)$. The problem is to come up with a reasonable definition of $\theta$. To do this we define, for $b=(x, \alpha, \beta)$ in $U(B)$, a two-dimensional sphere $S(b)$ containing $x$, orthogonal to the boundary sphere $S$ and with tangent plane at $x$ generated by $\alpha, \beta$. In other words, $S(b)$ is the totally geodesic 2 -plane determined by $\alpha$ and $\beta$. In the definition above we now choose $\theta$ to be the unique direction such that

$$
S(x, \alpha, \beta)=S\left(g_{t}(x, \alpha), \theta\right) \text {. }
$$

From the work of Hopf [2, p. 287 et seq.] we know that both flows can be defined on the identified space $\Omega_{1}=U(B) / \Gamma$, for any discrete group $\Gamma$, and that they both preserve the inherited measure $\mu$ on $\Omega_{1}$. Further, by extending Hopf's work from the case $\mu\left(\Omega_{1}\right)<\infty$ to the infinite-measure case (only minor modifications are necessary), we have

LEMMA 1. If $f$ is a bounded integrable function on $\Omega_{1}$, then $f$ is invariant under the geodesic flow if and only if it is invariant under the horocyclic flow.

In order to prove Theorem 1 it is sufficient to consider functions $f$ and $\lambda$ continuous with compact support on $\Omega$. Now note that if $f$ is a function defined on $\Omega$, we may define a function $f_{1}$ on $\Omega_{1}$ by projecting down to $T(B), U(B)$ and setting, for each $(x, \alpha, \beta), f_{1}(x, \alpha, \beta)=f(x, \alpha)$. For $f$ and $\lambda$ given as above, it is clear that

$$
K \int_{\Omega} f\left(g_{-t}(P)\right) \lambda(P) d m=\int_{\Omega_{1}} f_{1}\left(g_{-t}(Q)\right) \lambda_{1}(Q) d \mu,
$$

where $K$ is the solid angle subtended at the origin by the $(n-2)$-dimensional unit sphere. Thus, to prove Theorem 1 it suffices to show that, for bounded continuous functions $f, \lambda$ on $\Omega_{1}$ which are invariant under a change in the second direction and which are of compact support,

$$
\lim _{t \rightarrow \infty} \int_{\Omega_{1}} f\left(g_{-t}(Q)\right) \lambda(Q) d \mu=0 .
$$


At this point we use Hopf's idea [2, p. 286] and approximate the integral in the above limit by the integral

$$
\int_{\Omega_{1}}\left[\frac{1}{\delta} \int_{0}^{\delta} f\left(g_{-t}\left(h_{s}(Q)\right)\right) d s\right] \lambda(Q) d \mu .
$$

In view of our assumptions about $f$ and $\lambda$, it is not difficult to see that the convergence of this integral (as $\delta \rightarrow 0$ ) is uniform in $t$. Thus it remains to show that, for fixed $\delta>0$,

$$
\lim _{t \rightarrow \infty} \int_{\Omega_{1}}\left[\frac{1}{\delta} \int_{0}^{\delta} f\left(g_{-t}\left(h_{s}(Q)\right)\right) d s\right] \lambda(Q) d \mu=0 .
$$

A well-know relation between the geodesic and horocyclic flows is as follows [2, p. 285]. For any real $s$ and $t$,

$$
g_{t} \circ h_{s}=h_{s} \exp (-t) \circ g_{t}
$$

so the integral above becomes

$$
\int_{\Omega_{1}}\left[\frac{1}{\delta} \int_{0}^{\delta} f\left(h_{s \exp (t)}\left(g_{-t}(Q)\right)\right) d s\right] \lambda(Q) d \mu,
$$

and the change of variable $u=s \exp (t)$ yields

$$
\int_{\Omega_{1}}\left[\frac{1}{U} \int_{0}^{U} f\left(h_{u}\left(g_{-t}(Q)\right)\right) d u\right] \lambda(Q) d \mu,
$$

where $U=\delta \exp (t)$. Since $g_{t}$ preserves the measure $\mu$, this integral is equal to

$$
\int_{\Omega_{1}}\left[\frac{1}{U} \int_{0}^{U} f\left(h_{u}(Q)\right) d u\right] \lambda\left(g_{t}(Q)\right) d \mu .
$$

Now for fixed $t$ we apply the Cauchy-Schwarz inequality and noting that

$$
\int_{\Omega_{1}} \lambda^{2}\left(g_{t}(Q)\right) d \mu=\int_{\Omega_{1}} \lambda^{2}(Q) d \mu
$$

is bounded, it remains only to show that $(1 / U) \int_{0}^{U} f\left(h_{u}(Q)\right) d u$ converges, as $U \rightarrow \infty$, to zero in the sense of $L_{2}\left(\Omega_{1}, \mu\right)$ convergence. From the mean ergodic theorem we know that

$$
F(Q)=\lim _{U \rightarrow \infty} \frac{1}{U} \int_{0}^{U} f\left(h_{u}(Q)\right) d u
$$

exists, in the sense of $L_{2}\left(\Omega_{1}, \mu\right)$ convergence, is integrable and $h_{u}$ invariant. We know also, from the definition of $f$, that $F$ is invariant under a change in the second direction and, from the lemma, that $F$ is invariant under the geodesic flow.

Consider now two points $(x, \alpha, \beta)$ and $\left(x_{1}, \alpha_{1}, \beta_{1}\right)$ of $U(B)$ with the property that the geodesics determined by $(x, \alpha)$ and $\left(x_{1}, \alpha_{1}\right)$ have the same endpoint on the boundary sphere $S$. It is clear that we may move from $(x, \alpha, \beta)$ to $\left(x_{1}, \alpha_{1}, \beta_{1}\right)$ by a rotation of the second direction, a horocyclic flow, and a geodesic flow. In view of our comments above we may define a measurable $\Gamma$-invariant function $v$ on the boundary sphere $S$ by $v(\xi)=F(Q)$, where $Q$ lifts to $(x, \alpha, \beta)$ in $U(B)$ and $\xi$ is the endpoint of the geodesic determined by $(x, \alpha)$. 
Since $\Gamma$ is of divergence type it is known that the action of $\Gamma$ on $S$ is ergodic (see, for example, [1, p. 911]) - this is a fairly elementary result not to be confused with the far deeper theorem that a group of divergence type is ergodic on $S \times S$. Since $\Gamma$ is ergodic on $S$, the function $v$ above is a constant, and, thus, so is the function $F$. However, $F$ is also integrable on a space of infinite measure and is thus equal to zero almost everywhere. We have shown that

$$
\lim _{U \rightarrow \infty} \frac{1}{U} \int_{0}^{U} f\left(h_{u}(Q)\right) d u=0
$$

almost everywhere in $\Omega_{1}$, and this completes the proof of the theorem.

\section{REFERENCES}

1. Lars V. Ahlfors, Moebius transformations in several dimensions, Lecture Notes, School of Mathematics, Univ. of Minnesota, 1981.

2. Eberhard Hopf, Statistik der geodatischen Linien in Mannigfaltigkeiten negativer Krummung, Ber. Verh. Sachs. Akad. Wiss. Leipzig 91 (1939), 261-304.

3. P. J. Nicholls, Transitivity properties of Fuchsian groups, Canad. J. Math. 28 (1976), 805-814.

4. The orbital counting function for discrete groups of infinite volume, J. London Math. Soc. (2) 23 (1981), 460-468.

5. __ A lattice point problem in hyperbolic space, Michigan Math. J. 30 (1983), 273-287.

6. S. J. Patterson, Spectral theory and Fuchsian groups, Math. Proc. Cambridge Philos. Soc. 81 (1977), 59-75.

7. H. Shirakawa, On zero type of infinite measure preserving ergodic geodesic flow on a surface with constant negative curvature, Comment. Math. Univ. St. Paul 32 (1983), 1-13.

Department of Mathematics, Northern Illinois University, DeKalb, Illinois 60115 